\title{
Adaptive Sliding Control for a Class of Fractional Commensurate Order Chaotic Systems
}

\author{
Jian Yuan, ${ }^{1}$ Bao Shi, ${ }^{1}$ and Zhentao $\mathbf{Y u}^{2}$ \\ ${ }^{1}$ Institute of System Science and Mathematics, Naval Aeronautical and Astronautical University, Yantai 264001, China \\ ${ }^{2}$ Department of Navigation, Naval Submarine Academy, Qingdao 266001, China \\ Correspondence should be addressed to Jian Yuan; yuanjianscar@gmail.com
}

Received 13 June 2014; Accepted 22 September 2014

Academic Editor: Jocelyn Sabatier

Copyright (C) 2015 Jian Yuan et al. This is an open access article distributed under the Creative Commons Attribution License, which permits unrestricted use, distribution, and reproduction in any medium, provided the original work is properly cited.

\begin{abstract}
This paper proposes adaptive sliding mode control design for a class of fractional commensurate order chaotic systems. We firstly introduce a fractional integral sliding manifold for the nominal systems. Secondly we prove the stability of the corresponding fractional sliding dynamics. Then, by introducing a Lyapunov candidate function and using the Mittag-Leffler stability theory we derive the desired sliding control law. Furthermore, we prove that the proposed sliding manifold is also adapted for the fractional systems in the presence of uncertainties and external disturbances. At last, we design a fractional adaptation law for the perturbed fractional systems. To verify the viability and efficiency of the proposed fractional controllers, numerical simulations of fractional Lorenz's system and Chen's system are presented.
\end{abstract}

\section{Introduction}

In recent years, fractional calculus has attracted an increasing interest not only among mathematicians, but also among physicists and engineers. Fractional calculus is a generalization of the traditional integer order integration and differentiation to arbitrary noninteger (real or complex) order. In comparison with classical calculus, fractional-order derivatives and integrals provide more accurate modeling of dynamical systems possessing memory and hereditary properties [1, 2]. Various fractional dynamic models have been developed in rheology, viscoelasticity, electrochemistry, electromagnetism, and so forth [3].

To propose more efficient control design for fractional dynamic systems, fractional control provides a suitable way [2]. Fractional control has attracted interest in the control community since the first fractional controller, the CRONE controller, has been introduced and applied in various fields of control systems [4]. Other pioneering contributions on fractional control methodologies have been made, such as the TID controller, the fractional $P I^{\lambda} D^{\mu}$ controller [5], and the fractional lead-lag compensator [6]. Basic ideas and comparisons between the above four fractional control schemes have been addressed in [7]. More recently, several control methodologies for nonlinear fractional dynamic systems have been developed by combining fractional calculus and nonlinear control theory, such as the fractional sliding control [8-10], the fractional adaptive control [11-13], and the fractional optimal control $[1,14-16]$.

The works on fractional control go hand in hand with the stability of fractional differential equations (FDEs) and rely largely on their results [17]. Broad surveys in the stability issue involving fractional dynamic systems have been published. A complete and systematic picture of the state of the art in the stability of FDEs is provided in [18]. This review article covers the stability results in linear FDEs, nonlinear FDEs, and time delayed FDEs. There are two most important approaches to analyze the stability of nonlinear FDEs: one is to use the frequent distributed fractional integrator model [19], and the other is to use the Mittag-Leffler stability theorem [20]. The first approach relies on the concept of a fractional integration operator characterized by a continuous frequency distributed model. It involves two steps: firstly converting FDEs into exactly equivalent infinite dimensional ODEs, secondly applying the traditional indirect Lyapunov approach. Utilizing this approach, the stability of sliding 
dynamics and a fractional sliding control law for a novel class of fractional chaotic systems have been investigated in [21]. The stability and tracking convergence of fractional model reference adaptive control systems are analyzed in [13].

Analyzing the stability of nonlinear FDEs by using the second approach is usually a complicated task, because it is difficult to calculate the fractional-order derivative of the commonly used Lyapunov candidate function. Fortunately, a new property for Caputo fractional derivative is obtained in [22], providing an easy way to derive an inequality for the fractional-order derivative of the Lyapunov candidate function in the quadratic form, instead of directly calculating its fractional-order derivative. So it is potentially a convenient way to propose the stability analysis for nonlinear FDEs.

In this paper, our main objective is to propose adaptive sliding control design for a class of commensurate fractionalorder chaotic systems based on the newly discovered property of Caputo operator. For this end, we firstly introduce a fractional integral sliding manifold for these nominal systems. Next we prove the stability of the corresponding fractional sliding dynamics. Then, by introducing a quadratic Lyapunov control function and using the MittagLeffler stability theory we derive the desired control law. Furthermore, we prove that the proposed sliding manifold is also adapted for these fractional systems in the presence of uncertainties and external disturbances. At last, we investigate adaptive sliding control design for these perturbed systems.

The main contributions of this paper include the following: (1) a fractional integral sliding manifold is designed which is adapted not only for the nominal fractional systems but also for the perturbed systems; (2) the stability of the sliding dynamics corresponding to the nominal systems and the perturbed systems is proved; (3) a sliding control law and an adaptive law are designed, respectively, for the nominal systems and the perturbed systems.

The rest of the paper is organized as follows. Section 2 presents some basic definitions and theorems about fractional calculus and stability of fractional differential equations. Section 3 introduces a fractional integral sliding manifold and proposes the sliding control design for a class of nominal fractional-order chaotic systems. Section 4 investigates adaptive sliding control design for perturbed systems. In Section 5, numerical simulations of fractional Lorenz's system and Chen's system are presented to show the viability and efficiency of the proposed fractional controllers. Finally, the paper is concluded in Section 6.

\section{Basic Definitions and Preliminaries}

In this section we recall the definitions and several theorems in the fractional calculus. The most commonly used definitions of fractional derivatives are Grünwald-Letnikov, Riemann-Liouville, and Caputo definitions.
Definition 1. The Grünwald-Letnikov derivative definition of order $\alpha$ is described as

$$
{ }_{a} D_{t}^{\alpha} f(t)=\lim _{h \rightarrow 0} \frac{1}{h^{\alpha}} \sum_{j=0}^{[(t-a) / h]}(-1)^{j}\left(\begin{array}{c}
\alpha \\
j
\end{array}\right) f(t-j h),
$$

where $h$ is the time step.

Definition 2. The Riemann-Liouville derivative of order $\alpha$ is defined as

$$
{ }_{a} D_{t}^{\alpha} f(t)=\frac{1}{\Gamma(n-\alpha)} \frac{d^{n}}{d t^{n}} \int_{a}^{t} \frac{f(\tau) d \tau}{(t-\tau)^{\alpha-n+1}}
$$

for $n-1<\alpha<n$, where $\Gamma(\cdot)$ is Euler's Gamma function.

Definition 3. The Caputo definition of fractional derivative can be written as

$$
{ }_{a} D_{t}^{\alpha} f(t)=\frac{1}{\Gamma(n-\alpha)} \int_{a}^{t} \frac{f^{(n)}(\tau) d \tau}{(t-\tau)^{\alpha-n+1}}
$$

for $n-1<\alpha<n$.

Lemma 4 (fractional comparison principle [20]). Let $x(0)=$ $y(0)$ and $D^{\alpha} x(t) \geq D^{\alpha} y(t)$, where $0<\alpha<1$; then $x(t) \geq y(t)$.

Lemma 5 (Mittag-Leffler stability [20]). Let $x_{e q}=0$ be an equilibrium point of a fractional nonlinear system and $a$ domain containing the origin. Let $V(t, x(t)):[0, \infty) \times D \rightarrow$ $R^{+}$be a continuously differentiable function satisfying

$$
\begin{gathered}
V(t, x(t)) \geq \alpha(\|x\|), \\
D^{q} V(t, x(t)) \leq 0,
\end{gathered}
$$

where $\alpha(\cdot)$ is the class-K function, $x \in D$, and $0<q<1$. Then $x=0$ is globally stable.

Lemma 6 (see [22]). Let $x(t) \in R$ be a continuous and derivable function. Then, for any time instant $t \geq t_{0}$,

$$
\frac{1}{2}{ }_{t_{0}}^{C} D_{t}^{q} x^{2}(t) \leq x(t){ }_{t_{0}}^{C} D_{t}^{q} x(t), \quad \forall q \in(0,1) .
$$

\section{Sliding Control for the Nominal Fractional Chaotic Systems}

Consider a class of fractional-order chaotic systems described by the following fractional differential equations:

$$
\begin{aligned}
& D^{q_{1}} x=f(x, y, z)-\alpha x, \\
& D^{q_{2}} y=x g(x, y, z)-\beta y, \\
& D^{q_{3}} z=x h(x, y, z)-\gamma z,
\end{aligned}
$$

where $q_{1}, q_{2}, q_{3} \in(0,1)$ are fractional-orders, $x, y$, and $z$ are state variables, and $\alpha, \beta$, and $\gamma$ are nonnegative known constants. 
The above fractional system (6) is introduced by Yuan et al. [21]. It consists of 6 fractional chaotic systems, including the fractional-order Lorenz system, the fractional-order Chen system, the fractional-order Lü system, the fractionalorder Liu system, the fractional-order Lotka-Volterra system, and the fractional-order Rucklidge system.

In this paper, our main purpose is to propose the sliding control design for the commensurate ordered chaotic system, that is, the case when $q_{1}=q_{2}=q_{3}=q \in(0,1)$. Firstly, we introduce a fractional integral sliding surface which is different from the one in [21] and design sliding control law for the nominal fractional system (6) using the MittagLeffler stability theorem. Secondly, we consider system with uncertainties and external disturbances and propose adaptive sliding control design.

3.1. Sliding Surface Design. To propose the sliding control design for the nominal system (6), we introduce the following fractional integral sliding surface:

$$
s(t)=x(t)+{ }_{0} I_{t}^{q} \varphi(t)
$$

where $\varphi(t)=y g(x, y, z)+z h(x, y, z)+\alpha x$.

Taking its $q$ th order fractional derivative with respect to time leads to the following:

$$
D^{q} s(t)=D^{q} x(t)+\varphi(t) .
$$

3.2. Stability Analysis of Sliding Dynamics. Now we are about to prove that the system motion on the sliding manifold (i.e., sliding dynamics) satisfies our desired specifications; that is, once the trajectory of system (6) is steered to the sliding surface (7), the three state variables $x, y$, and $z$ will converge to zero asymptotically.

Let $D^{q} s(t)=0$; then we derive the following fractional sliding dynamics for the nominal system:

$$
\begin{gathered}
D^{q} x=-y g(x, y, z)-z h(x, y, z)-\alpha x, \\
D^{q} y=x g(x, y, z)-\beta y, \\
D^{q} z=x h(x, y, z)-\gamma z .
\end{gathered}
$$

For the sliding dynamics (9) a Lyapunov function is chosen as

$$
V_{1}(x, y, z)=\frac{1}{2} x^{2}+\frac{1}{2} y^{2}+\frac{1}{2} z^{2}
$$

By taking its $q$ th order fractional derivative with respect to time, using Lemma 6 , and inserting the three equations of system (9) into (10), one derives

$$
\begin{aligned}
D^{q} V_{1} & \leq x D^{q} x+y D^{q} y+z D^{q} z \\
& =x(-y g-z h-\alpha x)+y(x g-\beta y)+z(x h-\gamma z) \\
& =-\left(\alpha x^{2}+\beta y^{2}+\gamma z^{2}\right) .
\end{aligned}
$$

Denoting $\eta=\min \{\alpha, \beta, \gamma\}$, then inequality (11) becomes

$$
D^{q} V_{1} \leq-\eta V_{1}
$$

Inequality (12) satisfies the Mittag-Leffler stability theorem. This implies that the three state variables $x, y$, and $z$ tend to zero asymptotically. Up to this point, we come to the following statement.

Theorem 7. The fractional sliding dynamics (9) of the nominal fractional system (6) is asymptotically stable.

3.3. Sliding Control Design. To propose the sliding control design for the nominal system (6), we introduce the following control Lyapunov function:

$$
V_{2}(s)=\frac{1}{2} s^{2}
$$

Taking its $q$ th order fractional derivative with respect to time and inserting the first equation of system (6), one derives

$$
\begin{aligned}
D^{q} V_{2} \leq & s D^{q} s \\
= & s\left(D^{q} x+\varphi\right) \\
= & s[f(x, y, z)-\alpha x+u(t)+y g(x, y, z) \\
& +z h(x, y, z)+\alpha x] .
\end{aligned}
$$

Then the control law is constructed as

$$
\begin{aligned}
u(t)= & -f(x, y, z)-y g(x, y, z) \\
& -z h(x, y, z)-k_{1} \operatorname{sgn}(s)-k_{2} s .
\end{aligned}
$$

Substituting the control law (15) into (14) yields

$$
\begin{aligned}
D^{q} V_{2} & \leq s\left[-k_{1} \operatorname{sgn}(s)-k_{2} s\right] \\
& =-k_{1}|s|-k_{2} s^{2} \\
& \leq-k_{2} V_{2} .
\end{aligned}
$$

In terms of the Mittag-Leffler stability theorem, we obtain that $s \rightarrow 0$ and $x, y, z \rightarrow 0$.

Up to this point, we arrive at the following conclusion.

Theorem 8. The nominal fractional chaotic system (6) is asymptotically stabilized under the proposed sliding control law (15).

\section{Adaptive Sliding Control for the Perturbed System}

In this section, we are about to go further by considering the commensurate ordered chaotic system (6) in the presence 
of systematic uncertainties and external disturbances. It is described by the following fractional differential equations:

$$
\begin{aligned}
& D^{q} x=f(x, y, z)-\alpha x+\Delta f_{1}(x, y, z)+d_{1}(t), \\
& D^{q} y=x g(x, y, z)-\beta y+\Delta f_{2}(x, y, z)+d_{2}(t), \\
& D^{q} z=x h(x, y, z)-\gamma z+\Delta f_{3}(x, y, z)+d_{3}(t) .
\end{aligned}
$$

We assume that all the uncertainties and external disturbances are bounded; that is, $\left|\Delta f_{1}(x, y, z)\right|<\theta_{1},\left|d_{1}(t)\right|<\theta_{2}$, $\left|\Delta f_{2}(x, y, z)\right|<F_{2},\left|d_{2}(t)\right|<D_{2},\left|\Delta f_{3}(x, y, z)\right|<F_{3}$, and $\left|d_{3}(t)\right|<D_{3}$, where $\theta_{1}, \theta_{2}, F_{2}, D_{2}, F_{3}$, and $D_{3}$ are unknown nonnegative constants.

4.1. Stability Analysis of Sliding Dynamics of Perturbed Systems. Proceeding as before, we introduce the fractional integral sliding surface as in (7) and derive the following sliding dynamics:

$$
\begin{gathered}
D^{q} x=-y g(x, y, z)-z h(x, y, z)-\alpha x, \\
D^{q} y=x g(x, y, z)-\beta y+\Delta f_{2}(x, y, z)+d_{2}(t), \\
D^{q} z=x h(x, y, z)-\gamma z+\Delta f_{3}(x, y, z)+d_{3}(t) .
\end{gathered}
$$

To analyze the stability of the sliding dynamics (18), we introduce the following Lyapunov function:

$$
V_{3}(x, y, z)=\frac{1}{2} x^{2}+\frac{1}{2} y^{2}+\frac{1}{2} z^{2}
$$

Taking its $q$ th order fractional derivative with respect to time, using Lemma 6, and inserting the three equations of system (18) into (19), one derives

$$
\begin{aligned}
D^{q} V_{3} \leq & x D^{q} x+y D^{q} y+z D^{q} z \\
= & x(-y g-z h-\alpha x)+y\left(x g-\beta y+\Delta f_{2}+d_{2}\right) \\
& +z\left(x h-\gamma z+\Delta f_{3}+d_{3}\right) \\
= & -\left(\alpha x^{2}+\beta y^{2}+\gamma z^{2}\right)+y\left(\Delta_{2}+D_{2}\right)+z\left(\Delta_{3}+D_{3}\right) .
\end{aligned}
$$

We denote

$$
\rho=y\left(\Delta_{2}+D_{2}\right)+z\left(\Delta_{3}+D_{3}\right) .
$$

It is observed that $\rho$ is also bounded, since all the state variables of chaotic system have bounded amplitude [23]. As a result, we ultimately derive the following inequality:

$$
D^{q} V_{3} \leq-\eta V_{3}+\rho \text {. }
$$

Following the proof of Theorem 2 in [23], we conclude that the sliding dynamics (18) is globally stable.

Up to this point, we come to the following conclusion.
Theorem 9. The fractional sliding dynamics (18) of the fractional system (17) in the presence of system uncertainties and external disturbances is globally stable.

4.2. Adaptive Sliding Control Design. To propose the sliding control design for the perturbed system (17), we introduce the following Lyapunov function:

$$
V_{4}\left(x, y, z, \hat{\theta}_{1}, \widehat{\theta}_{2}\right)=\frac{1}{2}\left[s^{2}+\frac{1}{\mu_{1}}\left(\widehat{\theta}_{1}-\theta_{1}\right)^{2}+\frac{1}{\mu_{2}}\left(\widehat{\theta}_{2}-\theta_{2}\right)^{2}\right] .
$$

Taking its $q$ th order fractional derivative with respect to time and inserting the first equation of system (17) into (23), one obtains

$$
\begin{aligned}
D^{q} V_{4} \leq & s D^{q} s+\frac{1}{\mu_{1}}\left(\widehat{\theta}_{1}-\theta_{1}\right) D^{q} \widehat{\theta}_{1}+\frac{1}{\mu_{2}}\left(\widehat{\theta}_{2}-\theta_{2}\right) D^{q} \widehat{\theta}_{2} \\
= & s\left(D^{q} x+\varphi\right)+\frac{1}{\mu_{1}}\left(\widehat{\theta}_{1}-\theta_{1}\right) D^{q} \widehat{\theta}_{1} \\
& +\frac{1}{\mu_{2}}\left(\widehat{\theta}_{2}-\theta_{2}\right) D^{q} \widehat{\theta}_{2} \\
= & s\left[f-\alpha x+\Delta f_{1}+d_{1}+u(t)+y g+z h+\alpha x\right] \\
& +\frac{1}{\mu_{1}}\left(\widehat{\theta}_{1}-\theta_{1}\right) D^{q} \widehat{\theta}_{1}+\frac{1}{\mu_{2}}\left(\widehat{\theta}_{2}-\theta_{2}\right) D^{q} \widehat{\theta}_{2} .
\end{aligned}
$$

If we chose the control law as

$$
\begin{aligned}
u(t)= & -f(x, y, z)-y g(x, y, z)-z h(x, y, z) \\
& -\left(\hat{\theta}_{1}+\hat{\theta}_{2}+k_{1}\right) \operatorname{sgn}(s)-k_{2} s
\end{aligned}
$$

and the fractional adaptive law as

$$
\begin{aligned}
& D^{q} \widehat{\theta}_{1}=\mu_{1}|s|, \\
& D^{q} \widehat{\theta}_{2}=\mu_{2}|s|,
\end{aligned}
$$

then inequality (24) becomes

$$
\begin{aligned}
D^{q} V_{4} \leq & s\left[\Delta f_{1}+d_{1}-\left(\widehat{\theta}_{1}+\widehat{\theta}_{2}+k_{1}\right) \operatorname{sgn}(s)-k_{2} s\right] \\
& +\left(\widehat{\theta}_{1}-\theta_{1}\right)|s|+\left(\widehat{\theta}_{2}-\theta_{2}\right)|s| \\
= & \left(\Delta f_{1}+d_{1}\right) s-\left(\widehat{\theta}_{1}+\widehat{\theta}_{2}+k_{1}\right)|s| \\
& +\left(\widehat{\theta}_{1}-\theta_{1}\right)|s|+\left(\widehat{\theta}_{2}-\theta_{2}\right)|s|-k_{2} s^{2} \\
\leq & \left(\theta_{1}+\theta_{2}\right)|s|-\left(\widehat{\theta}_{1}+\widehat{\theta}_{2}+k_{1}\right)|s|+\left(\widehat{\theta}_{1}-\theta_{1}\right)|s| \\
& +\left(\widehat{\theta}_{2}-\theta_{2}\right)|s|-k_{2} s^{2} \\
= & -k_{1}|s|-k_{2} s^{2} \leq 0 .
\end{aligned}
$$


It is obvious that $D^{q} V_{4}\left(x, y, z, \widehat{\theta}_{1}, \widehat{\theta}_{2}\right)=0$ if and only if $s=0$. So we can conclude that $x, y, z \rightarrow 0, \widehat{\theta}_{1} \rightarrow \theta_{1}$, and $\widehat{\theta}_{2} \rightarrow \theta_{2}$.

Up to this point, we arrive at the following theorem.

Theorem 10. The fractional system (17) in the presence of system uncertainties and external disturbances is asymptotically stabilized under the proposed sliding control law (24) and the fractional adaptive law (25).

Remark 11. The control design proposed in this paper is different from those in $[9,21]$. Firstly, the fractional sliding surfaces are of different forms. Secondly, the stability of the fractional sliding dynamics is analyzed using the MittagLeffler stability theorem, instead of the continuous frequency distributed model (see [21]) or the traditional Lyapunov method (see [9]). More importantly, a stability analysis for the fractional sliding dynamics of the perturbed systems is given in this paper.

\section{Numerical Simulations}

To show the viability and efficiency of the proposed control design, we give two illustrative examples, fractionalorder Lorenz's system and fractional-order Chen's system. We utilize the proposed fractional integral sliding control technique to control the two nominal fractional chaotic systems. Furthermore, we apply the adaptive sliding control approach to control the fractional systems in the presence of system uncertainties and external disturbances.

Numerical simulations are implemented using the MAT$\mathrm{LAB}$ software. We utilize the algorithm for numerical calculation of fractional derivatives, which is introduced by Petrás in [24]. This algorithm takes advantage of the "Short-Memory" principle and derived from Grünwald-Letnikov Definition (1) based on the fact that three Definitions (1), (2), and (3) are equivalent for a wide class of functions.

5.1. Control of Fractional-Order Lorenz's System. Fractional Lorenz's system is described as

$$
\begin{gathered}
D^{q} x=-a(x-y), \\
D^{q} y=r x-y-x z, \\
D^{q} z=-b z+x y,
\end{gathered}
$$

where $a=10, r=28$, and $b=8 / 3$.

In terms of (7), the sliding surface is

$$
s(t)=x(t)+{ }_{0} I_{t}^{q} \varphi(t),
$$

where $\varphi(t)=a x(t)+r y(t)$.

Following (15), the control law is

$$
u(t)=-(a+r) y(t)-k_{1} \operatorname{sgn}(s)-k_{2} s .
$$

The performances of nominal closed-loop fractionalorder Lorenz's system are shown in Figures 1 and 2, under the proposed fractional integral sliding surface (29) and the sliding control law (30). Figure 1 shows the time response of the three state variables and the control input (30). Figure 2 shows the corresponding fractional integral sliding surface (29).

Parameters for the numerical simulations are specified, respectively, as follows: the fractional-order $q=0.993$ and the coefficients of control law $k_{1}=0.02, k_{2}=0.02$.

Next we investigate the control design for fractionalorder Lorenz's system in the presence of system uncertainties and external disturbances

$$
\begin{gathered}
D^{q} x=-a(x-y)+\Delta f_{1}+d_{1}+u(t), \\
D^{q} y=r x-y-x z+\Delta f_{2}+d_{2}, \\
D^{q} z=-b z+x y+\Delta f_{3}+d_{3} .
\end{gathered}
$$

To carry out numerical simulations, the system uncertainties and external disturbances in the above perturbed system are assumed, respectively, to be $\Delta f_{1}(x, y, z)=0.1+$ $0.1 \sin (\pi x), d_{1}(t)=0.1 \cos t, \Delta f_{2}(x, y, z)=0.1-0.2 \sin (\pi y)$, $d_{2}(t)=0.2 \sin (2 t), \Delta f_{3}(x, y, z)=0.1 x \cos (\pi z)$, and $d_{3}(t)=$ $0.1 \cos (\pi t)$.

In view of (25) and (26), the control law and the fractional adaptive law are, respectively,

$$
\begin{gathered}
u(t)=-(a+r) y-\left(\widehat{\theta}_{1}+\widehat{\theta}_{2}+k_{1}\right) \operatorname{sgn}(s)-k_{2} s, \\
D^{q} \widehat{\theta}_{1}=\mu_{1}|s| \\
D^{q} \widehat{\theta}_{2}=\mu_{2}|s| .
\end{gathered}
$$

Figures 3 and 4 illustrate the performances of controlled fractional Lorenz's system with uncertainties and external disturbances, under the proposed fractional integral sliding surface (29), the sliding control law (32), and the fractional adaptive law (33). The time responses of the state variables, the control input, and the estimation of uncertainties/external disturbances are depicted in Figure 3. The corresponding fractional integral sliding surface (29) is illustrated in Figure 4.

Parameters for the numerical simulations are specified, respectively, as follows: the fractional-order $q=0.993$, the coefficients of control law $k_{1}=0.02, k_{2}=0.02$, the coefficients of adaptive law $\mu_{1}=0.03, \mu_{2}=0.02$, and the initial conditions of the adaptive parameters $\theta_{1}(0)=\theta_{2}(0)=$ 0.2 .

5.2. Control of Fractional-Order Chen's System. Fractionalorder Chen's system is described as

$$
\begin{gathered}
D^{q_{1}} x=a(y-x), \\
D^{q_{2}} y=(c-a) x-x z+c y+u, \\
D^{q_{3}} z=x y-b z .
\end{gathered}
$$




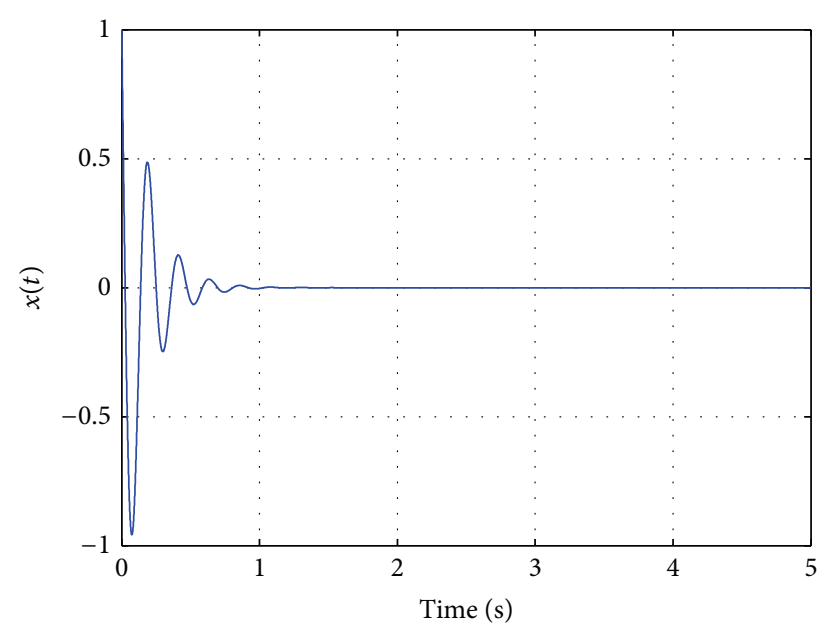

(a)

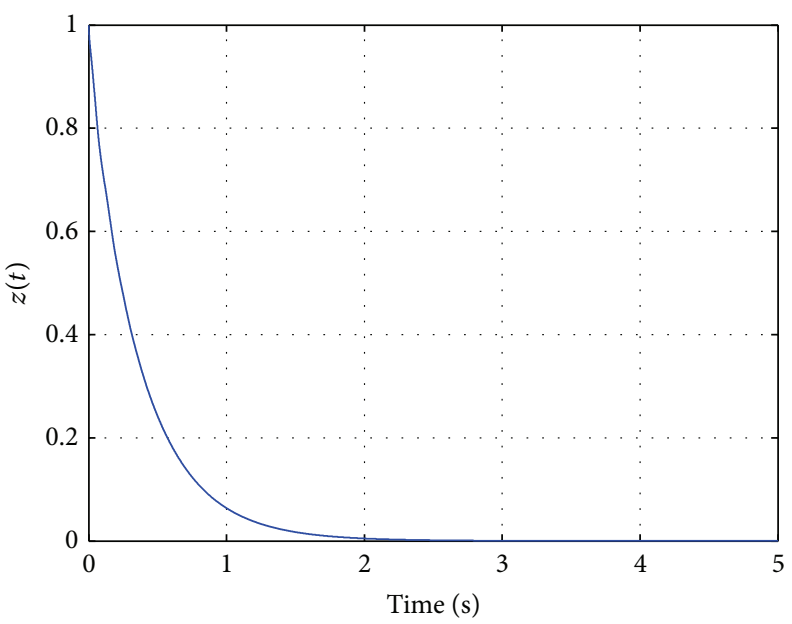

(c)

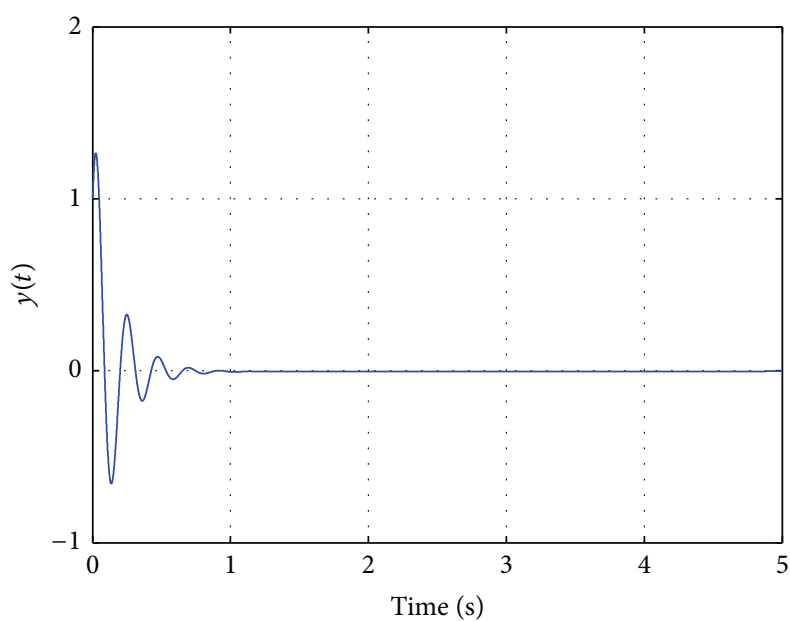

(b)

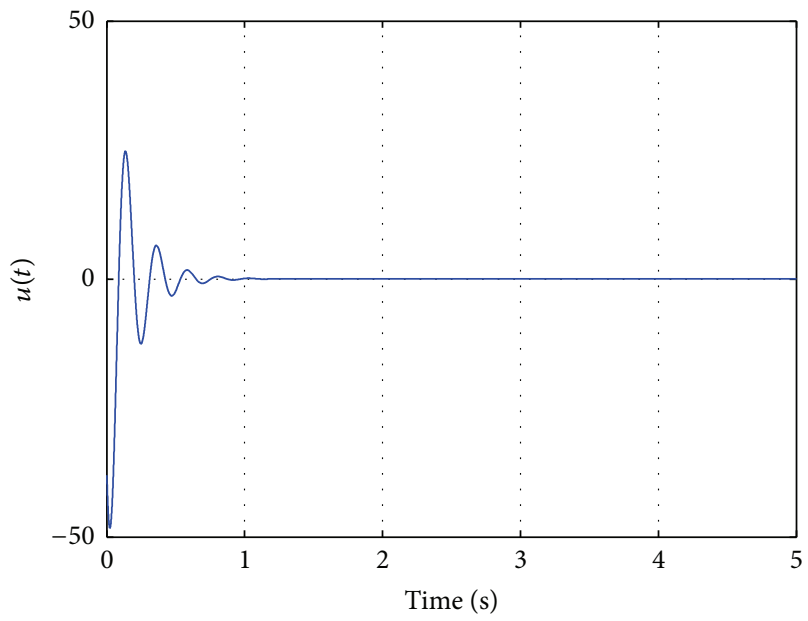

(d)

FIGURE 1: Fractional sliding control of nominal fractional-order Loren's system with time step $h=0.0005$ : (a) the $x$ - $t$ space; (b) the $y$ - $t$ space; (c) the $z$ - $t$ space; (d) the $u$ - $t$ space.

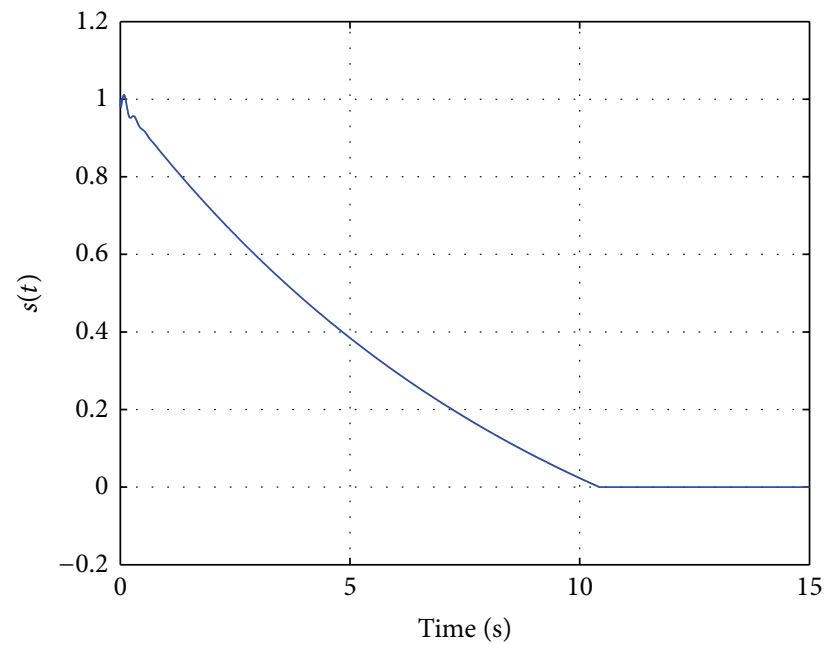

FIGURE 2: The time response of the sliding manifold (29).
In terms of (7), the sliding surface is

$$
s(t)=y(t)+{ }_{0} I_{t}^{q} \varphi(t)
$$

where $\varphi(t)=a x(t)+c y(t)+x(t) z(t)$.

Following (15), the control law is

$$
u(t)=-c x-2 c y-k_{1} \operatorname{sgn}(s)-k_{2} s .
$$

The performances of nominal closed-loop fractionalorder Chen's system are shown in Figures 5 and 6, under the proposed fractional integral sliding surface (35) and the sliding control law (36). Figure 5 shows the time response of the three state variables and the control input (36). Figure 6 shows the corresponding fractional integral sliding surface (35). 


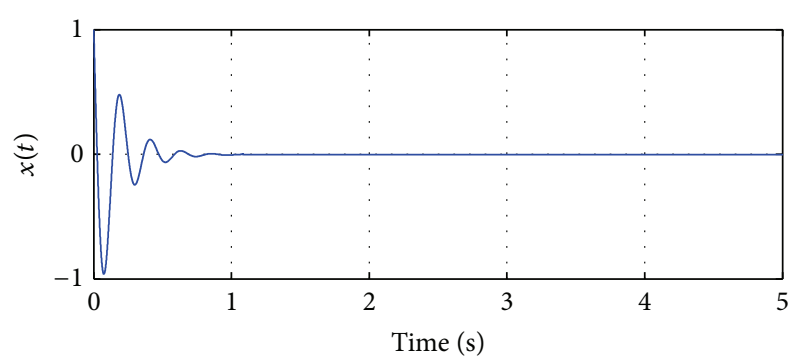

(a)

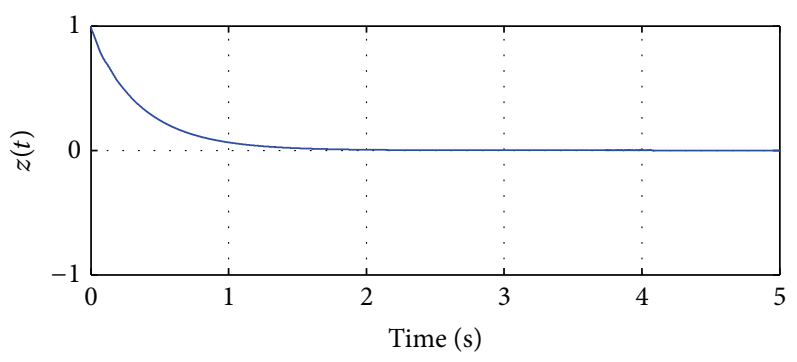

(c)

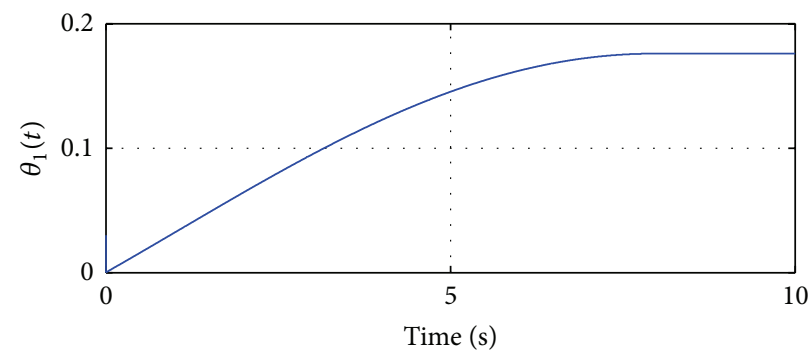

(e)

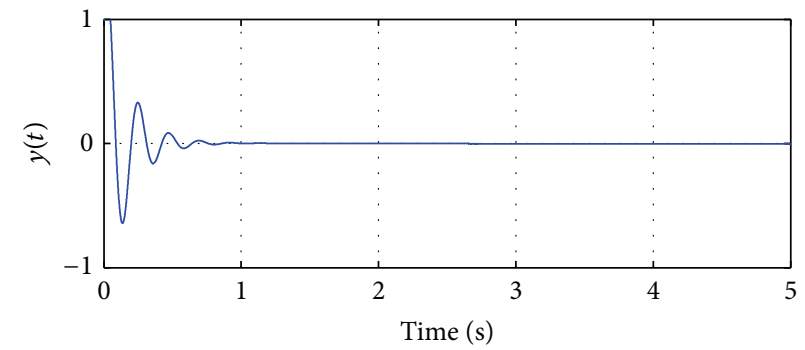

(b)

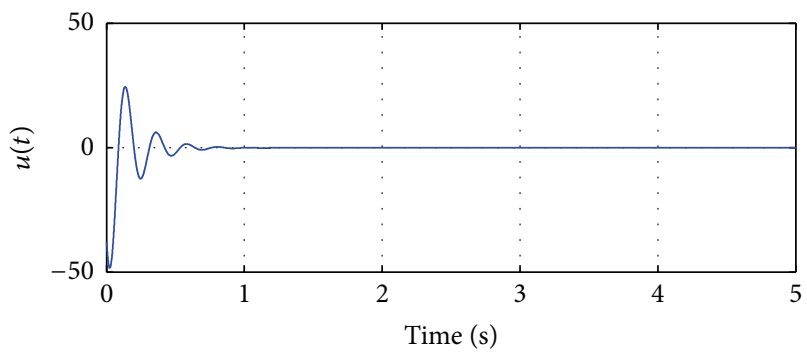

(d)

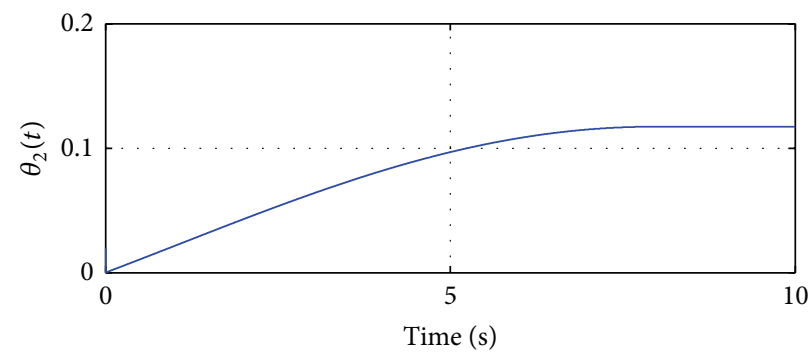

(f)

FIGURE 3: Adaptive sliding control of fractional-order Loren's system with dynamics uncertainties and external disturbances: (a) the $x$ - $t$ space; (b) the $y$ - $t$ space; (c) the $z$ - $t$ space; (d) the $u$ - $t$ space; (e) online estimate of $\theta_{1}$; (f) online estimate of $\theta_{2}$.

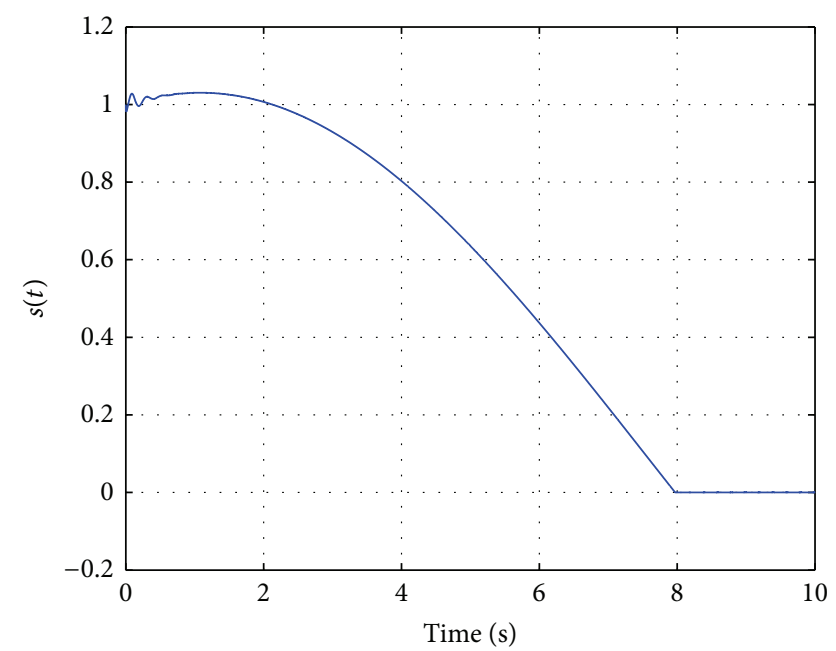

FIGURE 4: The time response of the sliding manifold (29).

Parameters for the numerical simulations are specified, respectively, as follows: the fractional-order $q=0.9$ and the coefficients of control law $k_{1}=0.1, k_{2}=0.1$.
Next we propose the adaptive sliding control design for fractional-order Chen's system in the presence of uncertainties and external disturbances which are added to the three equations. The control input is added to the second equation. Then the perturbed system to be controlled reads as

$$
\begin{gathered}
D^{q_{1}} x=a(y-x)+\Delta f_{1}+d_{1}, \\
D^{q_{2}} y=(c-a) x-x z+c y+\Delta f_{2}+d_{2}+u(t), \\
D^{q_{3}} z=x y-b z+\Delta f_{3}+d_{3} .
\end{gathered}
$$

To carry out numerical simulations, the system uncertainties and external disturbances in the above perturbed system are assumed, respectively, as $\Delta f_{1}(x, y, z)=0.1 \cos (x y)$, $d_{1}(t)=0.2 \sin (3 t), \Delta f_{2}(x, y, z)=0.2-0.1 \sin (\pi x), d_{2}(t)=$ $0.1 \cos t, \Delta f_{3}(x, y, z)=0.1 \sin (x y)$, and $d_{3}(t)=-0.1$ $\cos (5 t)$. 


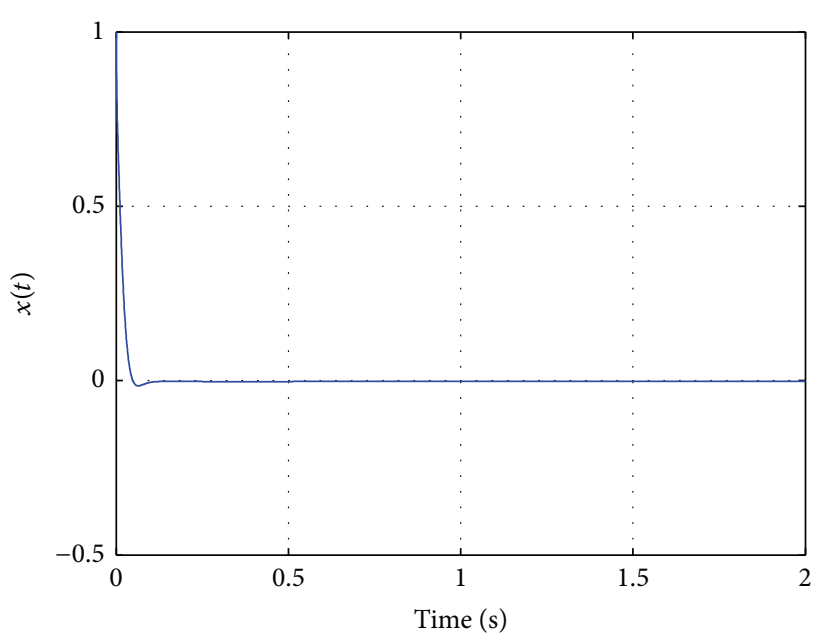

(a)

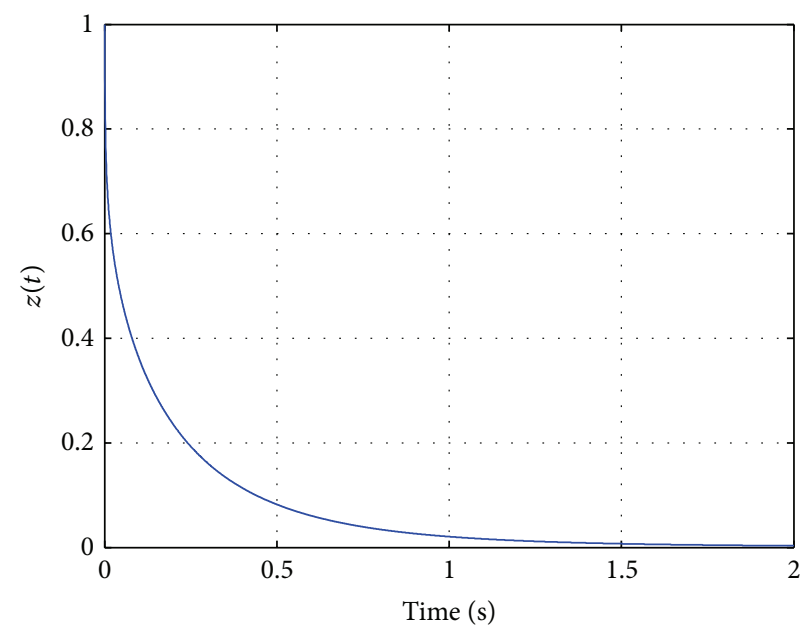

(c)

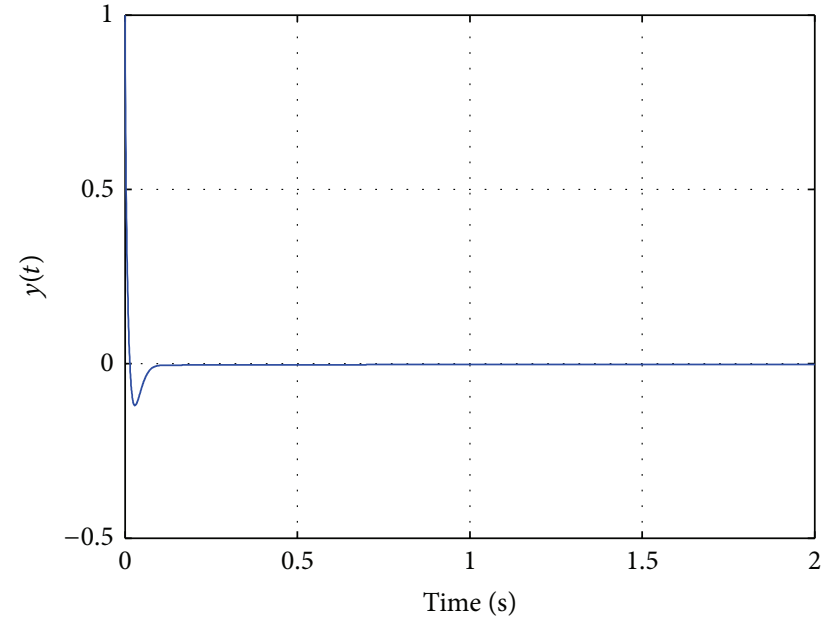

(b)

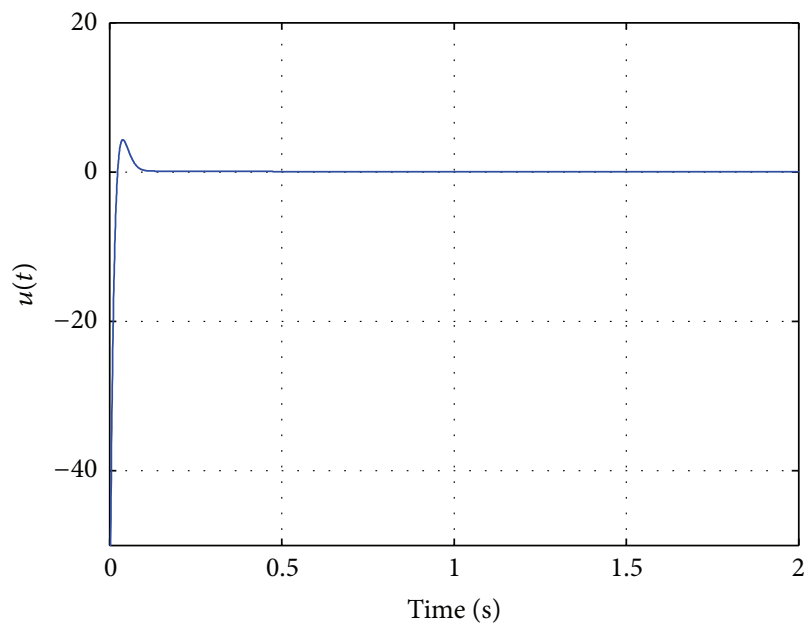

(d)

FIGURE 5: Sliding control of nominal fractional-order Chen's system: (a) the $x-t$ space; (b) the $y$ - $t$ space; (c) the $z$ - $t$ space; (d) the $u$ - $t$ space.

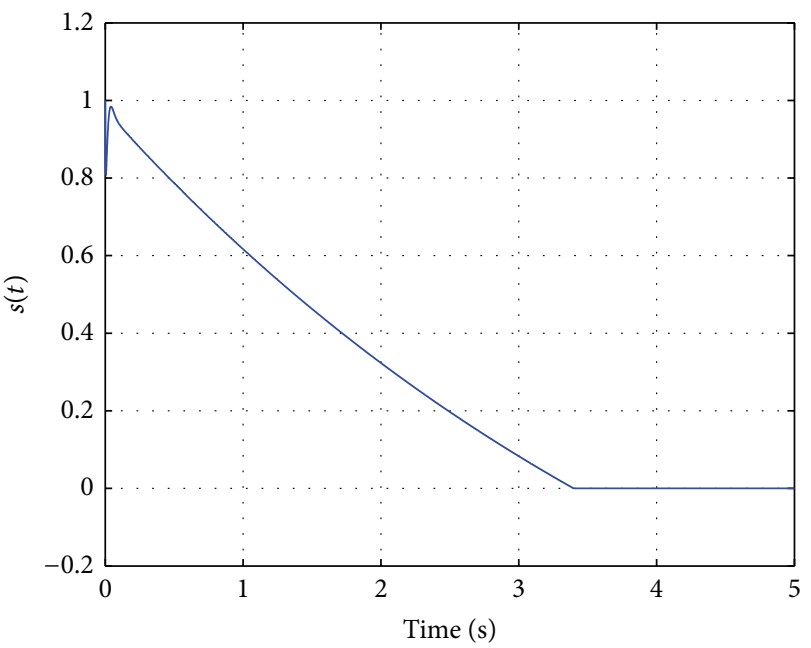

Figure 6: The time response of the sliding manifold (35).
In terms of (25) and (26), the control law and factional adaptive law are selected, respectively, as

$$
\begin{gathered}
u(t)=-c x-2 c y-\left(\widehat{\theta}_{1}+\widehat{\theta}_{2}+k_{1}\right) \operatorname{sgn}(s)-k_{2} s, \\
D^{q} \widehat{\theta}_{1}=\mu_{1}|s|, \\
D^{q} \widehat{\theta}_{2}=\mu_{2}|s| .
\end{gathered}
$$

Figures 7 and 8 illustrate the performances of controlled fractional-order Chen's system with uncertainties and external disturbances, under the proposed fractional integral sliding surface (35), the sliding control law (38), and the fractional adaptive law (39). The time responses of the state variables, the control input (38), and the estimation of uncertainties/external disturbances are depicted in Figure 7; the corresponding fractional integral sliding surface (35) is illustrated in Figure 8. 


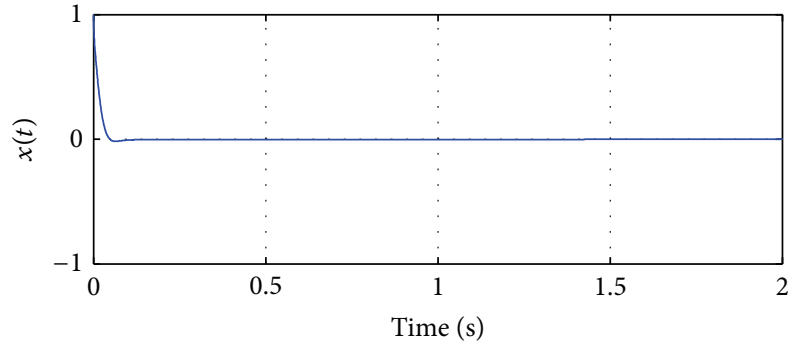

(a)

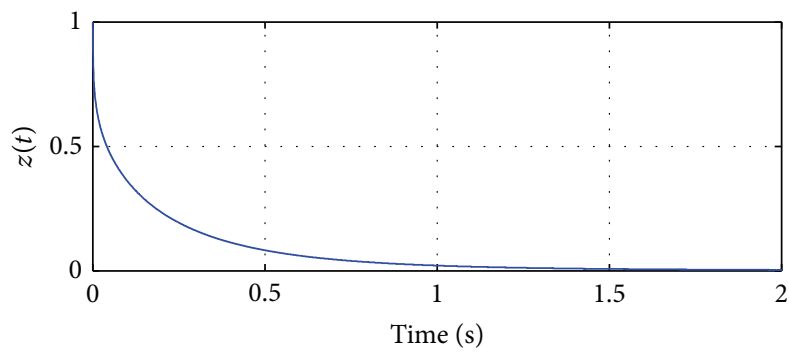

(c)

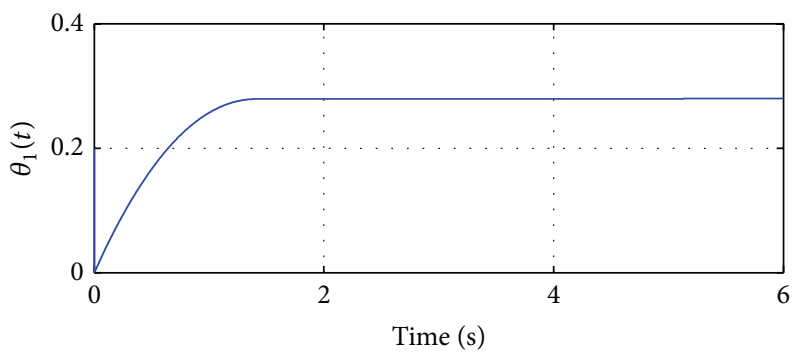

(e)

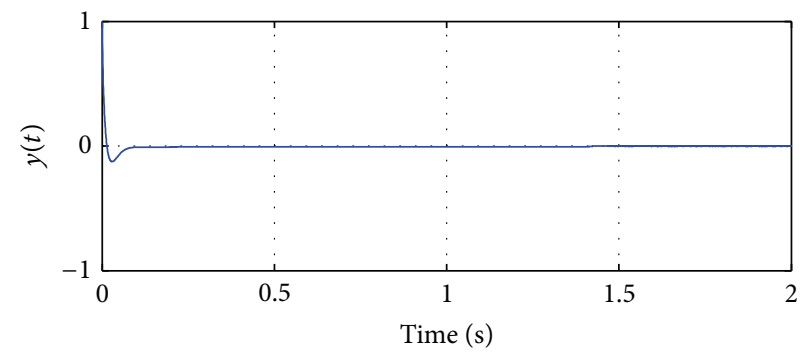

(b)

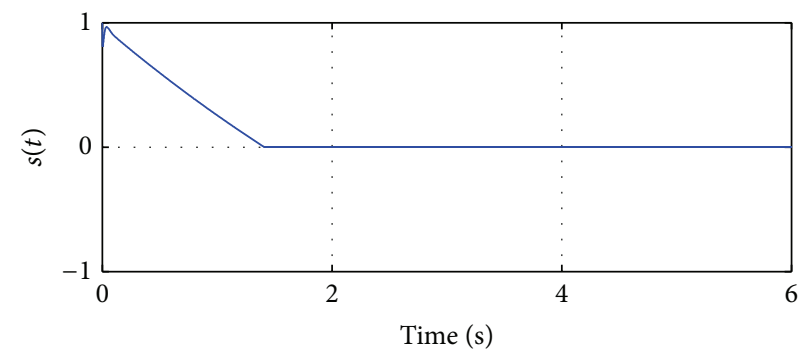

(d)

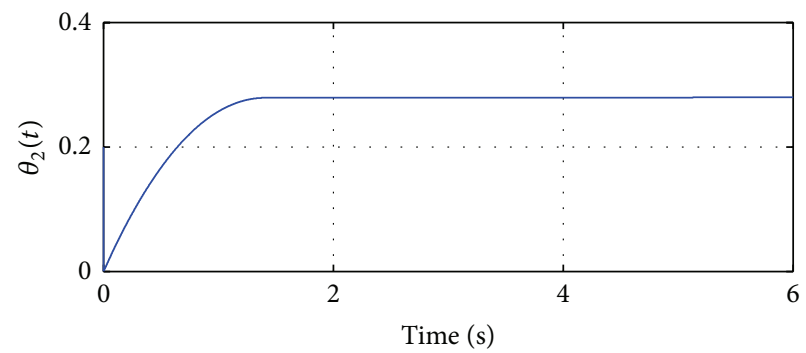

(f)

FIGURE 7: Adaptive sliding control of fractional-order Chen's system with dynamics uncertainties and external disturbances: (a) the $x$ - $t$ space; (b) the $y$ - $t$ space; (c) the $z$ - $t$ space; (d) the $u$ - $t$ space; (e) online estimate of $\theta_{1}$; (f) online estimate of $\theta_{2}$.

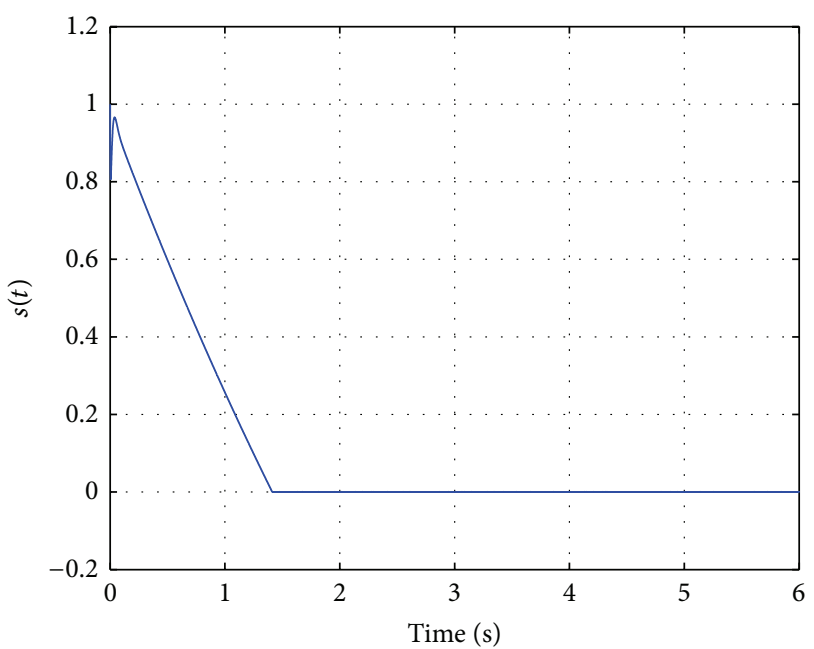

FIGURE 8: The time response of the sliding manifold (35).

Parameters for the numerical simulations are specified, respectively, as follows: the fractional-order $q=0.9$, the coefficients of control law $k_{1}=0.5, k_{2}=0.1$, the coefficients of adaptive law $\mu_{1}=0.2, \mu_{2}=0.2$, and the initial conditions of the adaptive parameters $\theta_{1}(0)=\theta_{2}(0)=0.2$.

\section{Conclusions}

In this paper, a fractional sliding controller and an adaptive sliding controller have been, respectively, designed for a class of commensurate fractional-order chaotic systems and the perturbed ones. Firstly, a fractional integral sliding manifold for the nominal systems has been introduced. Secondly, the stability of the corresponding fractional sliding dynamics has been proved. Then, by introducing a Lyapunov candidate function and using the Mittag-Leffler stability theory, a desired sliding control law has been obtained. Furthermore, the proposed sliding manifold has been proved to be adapted for the fractional systems in the presence of uncertainties and external disturbances. At last, an adaptation law for the perturbed fractional systems has been designed. Numerical simulations of fractional Lorenz's system and Chen's system are presented to verify the viability and efficiency of the proposed fractional controllers. 


\section{Conflict of Interests}

The authors do not have any possible conflict of interests.

\section{Acknowledgment}

This study was supported by a grant from the Natural Science Foundation of the Province Shandong of China (ZR2014AM006).

\section{References}

[1] O. P. Agrawal, "A general formulation and solution scheme for fractional optimal control problems," Nonlinear Dynamics, vol. 38, no. 1-4, pp. 323-337, 2004.

[2] I. Podlubny, Fractional Differential Equations: An Introduction to Fractional Derivatives, Fractional Differential Equations, to Methods of Their Solution and Some of Their Applications, Academic Press, 1998.

[3] K. Diethelm, The Analysis of Fractional Differential Equations: An Application-Oriented Exposition Using Differential Operators of Caputo Type, vol. 2004 of Lecture Notes in Mathematics, Springer, Berlin, Germany, 2010.

[4] A. Oustaloup, X. Moreau, and M. Nouillant, "The CRONE suspension," Control Engineering Practice, vol. 4, no. 8, pp. 1101$1108,1996$.

[5] I. Podlubny, "Fractional-order systems and $P I^{\lambda} D^{\mu}$-controllers," IEEE Transactions on Automatic Control, vol. 44, no. 1, pp. 208214, 1999.

[6] H.-F. Raynaud and A. Zergaïnoh, "State-space representation for fractional order controllers," Automatica, vol. 36, no. 7, pp. 1017-1021, 2000.

[7] D. Xue and Y. Q. Chen, "A comparative introduction of four fractional order controllers," in Proceedings of the 4th World Congress on Intelligent Control and Automation, pp. 3228-3235, Shanghai, China, June 2002.

[8] A. Razminia and D. Baleanu, "Complete synchronization of commensurate fractional order chaotic systems using sliding mode control," Mechatronics, vol. 23, no. 7, pp. 873-879, 2013.

[9] C. Yin, S. Dadras, S.-M. Zhong, and Y. Chen, "Control of a novel class of fractional-order chaotic systems via adaptive sliding mode control approach," Applied Mathematical Modelling, vol. 37, no. 4, pp. 2469-2483, 2013.

[10] S. Dadras and H. R. Momeni, "Fractional terminal sliding mode control design for a class of dynamical systems with uncertainty," Communications in Nonlinear Science and Numerical Simulation, vol. 17, no. 1, pp. 367-377, 2012.

[11] B. M. Vinagre, I. Petráš, I. Podlubny, and Y. Q. Chen, "Using fractional order adjustment rules and fractional order reference models in model-reference adaptive control," Nonlinear Dynamics, vol. 29, no. 1-4, pp. 269-279, 2002.

[12] S. Ladaci and A. Charef, "On fractional adaptive control," Nonlinear Dynamics, vol. 43, no. 4, pp. 365-378, 2006.

[13] B. Shi, J. Yuan, and C. Dong, "On fractional model reference adaptive control," The Scientific World Journal, vol. 2014, Article ID 521625, 8 pages, 2014.

[14] Z. D. Jelicic and N. Petrovacki, "Optimality conditions and a solution scheme for fractional optimal control problems," Structural and Multidisciplinary Optimization, vol. 38, no. 6, pp. 571-581, 2009.
[15] R. Kamocki, "On the existence of optimal solutions to fractional optimal control problems," Applied Mathematics and Computation, vol. 235, pp. 94-104, 2014.

[16] A. B. Malinowska and D. F. Torres, Introduction to the Fractional Calculus of Variations, Imperial College Press, London, UK, 2012.

[17] A. G. Butkovskii, S. S. Postnov, and E. A. Postnova, "Fractional integro-differential calculus and its control-theoretical applications. II. Fractional dynamic systems: modeling and hardware implementation," Automation and Remote Control, vol. 74, no. 5, pp. 725-749, 2013.

[18] C. P. Li and F. R. Zhang, "A survey on the stability of fractional differential equations," European Physical Journal: Special Topics, vol. 193, no. 1, pp. 27-47, 2011.

[19] J. C. Trigeassou, N. Maamri, J. Sabatier, and A. Oustaloup, "A Lyapunov approach to the stability of fractional differential equations," Signal Processing, vol. 91, no. 3, pp. 437-445, 2011.

[20] Y. Li, Y. Chen, and I. Podlubny, "Stability of fractional-order nonlinear dynamic systems: lyapunov direct method and generalized Mittag-Leffler stability," Computers \& Mathematics with Applications, vol. 59, no. 5, pp. 1810-1821, 2010.

[21] J. Yuan, B. Shi, and W. Ji, "Adaptive sliding mode control of a novel class of fractional chaotic systems," Advances in Mathematical Physics, vol. 2013, Article ID 576709, 13 pages, 2013.

[22] N. Aguila-Camacho, M. A. Duarte-Mermoud, and J. A. Gallegos, "Lyapunov functions for fractional order systems," Communications in Nonlinear Science and Numerical Simulation, vol. 19, no. 9, pp. 2951-2957, 2014.

[23] M. R. Faieghi, H. Delavari, and D. Baleanu, "A note on stability of sliding mode dynamics in suppression of fractional-order chaotic systems," Computers \& Mathematics with Applications, vol. 66, no. 5, pp. 832-837, 2013.

[24] I. Petráš, Fractional-Order Nonlinear Systems: Modeling, Analysis and Simulation, Springer, 2011. 


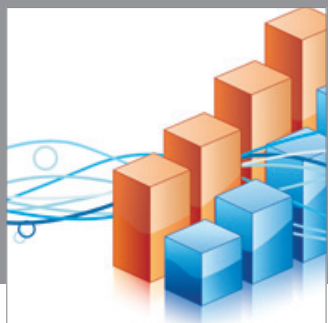

Advances in

Operations Research

mansans

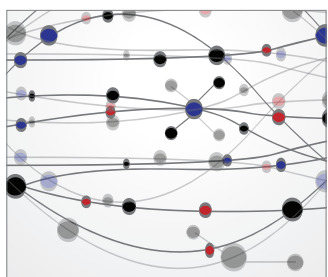

The Scientific World Journal
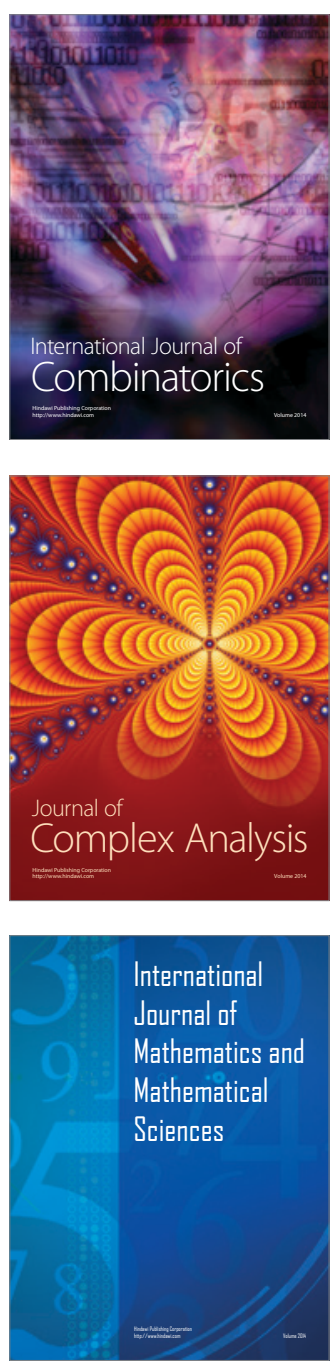
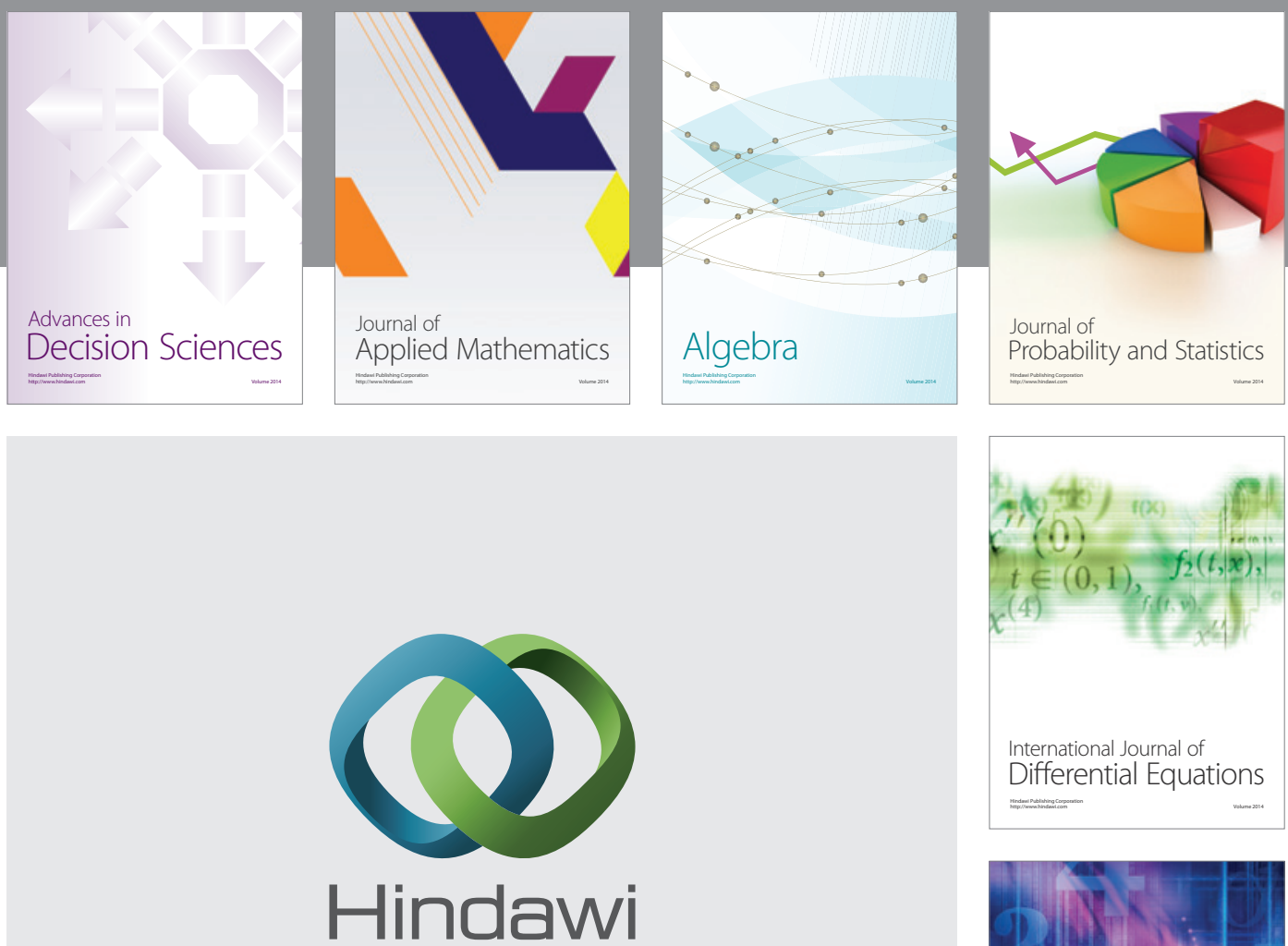

Submit your manuscripts at http://www.hindawi.com
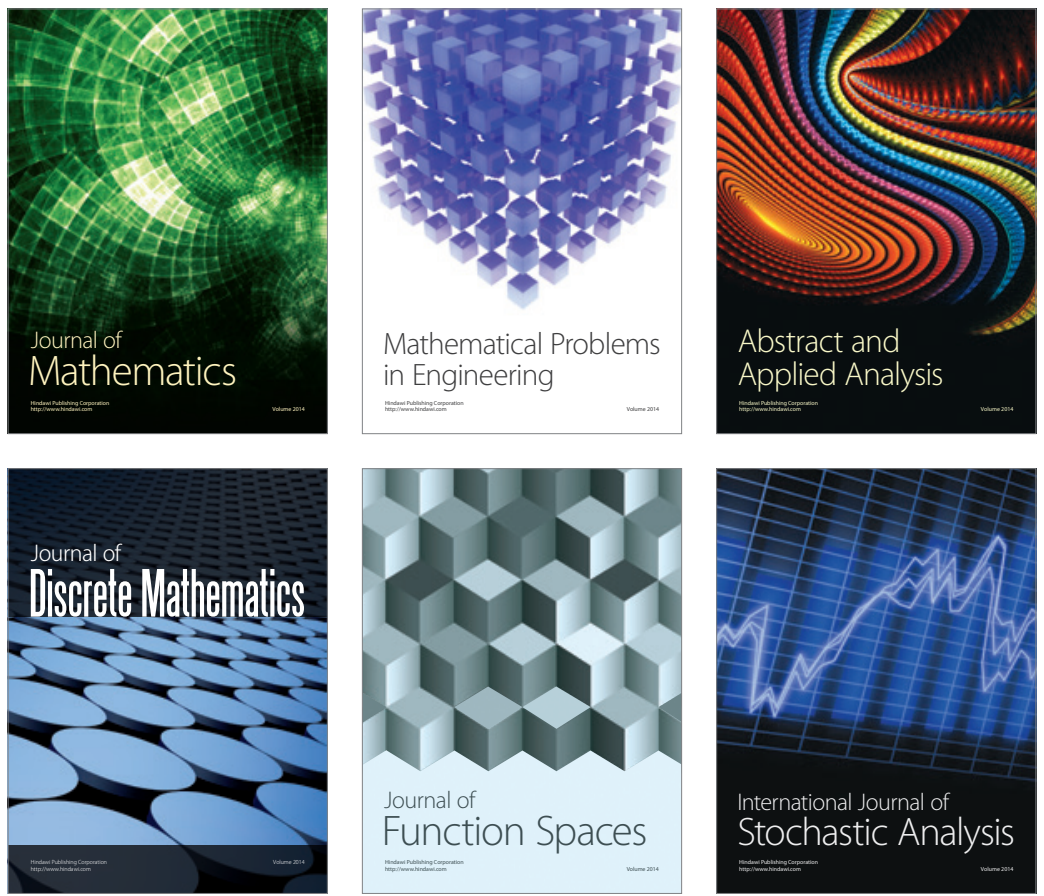

Journal of

Function Spaces

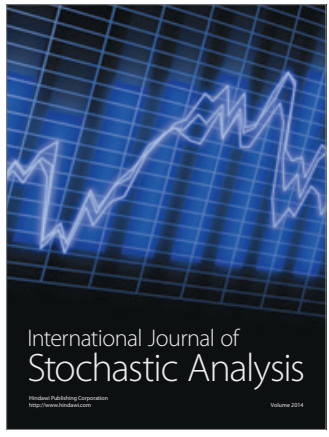

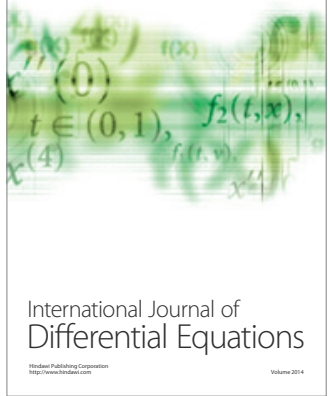
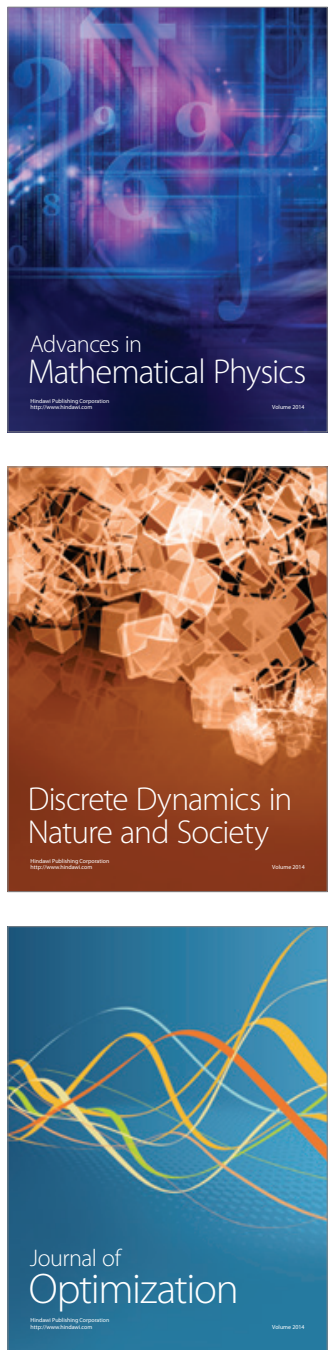